\title{
Tratamiento de la incontinencia urinaria de esfuerzo con Biofeedback perineal con electrodos de superficie
}

\author{
Lorenzo Gómez MF, Silva Abuín JM, García Criado FJ*, Geanini Yagüez A**, Urrutia Avisrror M. \\ Servicio y Cátedra de Urología. *Departamento de Cirugía. **Servicio de Rehabilitación. \\ Hospital Universitario de Salamanca. Universidad de Salamanca.
}

Actas Urol Esp. 2008;32(6):629-636

\section{RESUMEN}

TRATAMIENTO DE LA INCONTINENCIA URINARIA DE ESFUERZO CON BIOFEEDBACK PERINEAL CON ELECTRODOS DE SUPERFICIE

Objetivo: Se analiza el resultado del tratamiento de los músculos perineales mediante biofeedback (BFB) con electrodos de superficie en mujeres diagnosticadas de incontinencia urinaria de esfuerzo (IUE). Además se compara con la reeducación de la musculatura del suelo pélvico (RMSP) más electroestimulación vaginal (EEV).

Métodos: 85 mujeres diagnosticadas de IUE; de edades comprendidas entre 42 y 74 años, se dividieron en: Grupo 1 (N=50): tratadas mediante un programa de BFB perineal con electrodos de superficie sin electroestimulación y Grupo 2 (N=35): tratadas con RMSP con EEV. Se realizaron dos sesiones semanales de 30 minutos cada una durante 10 semanas. Se evaluaron los resultados mediante el estudio de los cambios en los índices internacionales de la incontinencia urinaria (IU-5 y el índice ICIQ-SF) y el índice de calidad de vida derivada de los síntomas urinarios (cuestionario de salud King`s). Se emplearon la t de Student y el test exacto de Fisher, p < 0,05 se aceptó como significación estadística.

Resultados: La edad de ambos grupos fue comparable. $84 \%$ y $80 \%$ de pacientes en los grupos 1 y 2 mejoraron respectivamente, entendiendo por mejora la no existencia de episodios de incontinencia y la ausencia de necesidad de usar absorbentes. No hubo diferencia en la mejora de la incontinencia entre ambos grupos. En el Grupo 1 la mejora se produjo en el 50\% de pacientes en la semana 4 , y en el $84 \%$ en la semana 10. En el Grupo 2 la mejoría ocurrió en el 71,42\% de pacientes en la semana 4 y en el $80 \%$ de pacientes en la semana 10 . Ocho pacientes del Grupo $2(22,85 \%)$ presentaron efectos secundarios locales. La mejora en la calidad de vida en ambos grupos arrojó resultados similares.

Conclusión: El éxito del tratamiento de la incontinencia urinaria de esfuerzo grados 1 y 2 mediante un programa de biofeedback perineal con electromiografía con electrodos de superficie sin electroestimulación es similar o superior a los publicados en tratamientos más invasivos. El tratamiento con ejercicios del suelo pélvico con electroestimulación vaginal puede conseguir resultados similares, aunque puede presentar efectos secundarios. Ambos tratamientos conservadores son eficaces y factibles.

Palabras claves: Incontinencia urinaria de esfuerzo. Suelo pélvico. Biofeedback perineal.

\section{ABSTRACT}

TREATMENT OF STRESS URINARY INCONTINENCE WITH PERINEAL BIOFEEDBACK BY USING SUPERFICIAL ELECTRODES

Purpose: We analyze the pelvic floor muscles treatment outcomes by using biofeedback (BFB) with electromyography with superficial electrodes in women diagnosed as having stress urinary incontinence (SUI). Besides, we compare this treatment with pelvic floor muscle exercises (PFME) plus vaginal electrostimulation.

Methods: 85 women with stress urinary incontinence, aged 42 - 74 years. We divided the patients in two groups: Group 1 (N=50): This patients carry out a perineal biofeedback with superficial electrodes without electrostimulation, and Group 2 (N=35): This patients were treated with pelvis floor muscle exercices and vaginal electrostimulation. All patients carry out two session per week (of 30 minutes each one) during ten weeks. We assess the outcomes through international urinary incontinence questionnaires (IU-5 and ICIQ-SF) and urinary incontinence related quality of life test (King s questionnaire). Student t-test and Fisher Exact test were used, p $<0.05$ was considered statistically significant.

Results: No difference was found in the age average of both groups. $84 \%$ of patients of group 1 and $80 \%$ of patients of group 2 were cured with the treatment. We assumed they were cured when incontinence episodes not happened or they do not need to use absorbent materials. In the Group 1, 50\% of patients in the fourth week and 84\% in the tenth week were cured. In the Group 2, 71,42\% of patients in the fourth week and $80 \%$ in the tenth week were cured. In the Group 2, eight patients $(22,85 \%)$ complained side effects. Both groups improved the quality of life similarly.

Conclusion: Grade 1 and grade 2 stress urinary incontinence treatment by using perineal biofeedback with superficial electrodes electromyography is better or similar to more invasive treatments. Also pelvic floor muscle exercices plus vaginal electrostimulation have good outcomes although some patients complain side effects. Both conservative treatments are effective and feasible. 
$\mathrm{L}$ a incontinencia urinaria de esfuerzo (IUE) es la pérdida involuntaria de orina por la uretra, sincrónicamente con esfuerzos o ejercicios, que supongan un incremento de la presión abdominal: estornudo, tos, actividad física, cambios posturales, etc ${ }^{1-2}$. En nuestro medio, hasta el 18\% de mujeres menores de 29 años han presentado algún síntoma, el $37 \%$ de mujeres de entre 35 y 54 años y el 39\% de las mayores de 55 años padecen $\mathrm{IUE}^{3}$.

El sistema de soporte uretral está formado por cuatro componentes: 1) Cara anterior vaginal; 2) Fascia endopélvica; 3) Arco tendíneo de la fascia pélvica y 4) Músculos elevadores del ano ${ }^{4}$.

Los músculos superficiales perineales son los músculos isquiocavernoso, bulbocavernoso, esfínter uretral externo, transverso superficial del periné y esfínter anal externo. El diafragma pélvico está constituido por los músculos elevadores -pubocoxígeo, puborectal, iliocoxígeo- y los músculos coxígeos y el diafragma urogenital sostenido por el músculo transverso profundo (Fig 1) .

La cuña perineal, también llamada centro tendinoso o rafe medio del periné, está constituida por elementos musculares lisos y tendinosos estriados, que van desde el pubis al isquion. Es un elemento importante en la eficacia del sistema de continencia urinaria $^{5-6}$. La cuña perineal se relaciona con la vagina, a la que rodea por detrás, con la curvatura perineal del recto y con los elevadores del ano (fibras musculares lisas insertadas en la aponeurosis).

El sistema de cierre esfinteriano está constituido por el músculo estriado uretral, el músculo liso uretral y los elementos vasculares de la submucosa ${ }^{4}$.

Clásicamente se aceptaban dos tipos de IU: La incontinencia urinaria de esfuerzo (IUE) debida a la pérdida del ángulo uretro vesical o al fallo intrínseco del esfinter uretral externo, y la incontinencia urinaria de urgencia (IUU) debida a contracciones no inhibidas del detrusor.

La actual teoría integral de la IU explica que la laxitud de la vagina y de los ligamentos de apoyo, altera el reflejo de la tensión mantenida, lo que impide una correcta distensibilidad para la función de almacenamiento (IUU) y una correcta plasticidad y tensión para mantener la continencia con la correcta transmisión de presiones, así como también dificulta la embudización y morfología de las estructuras para la micción y la continencia respectivamente (IUE) ${ }^{7}$.

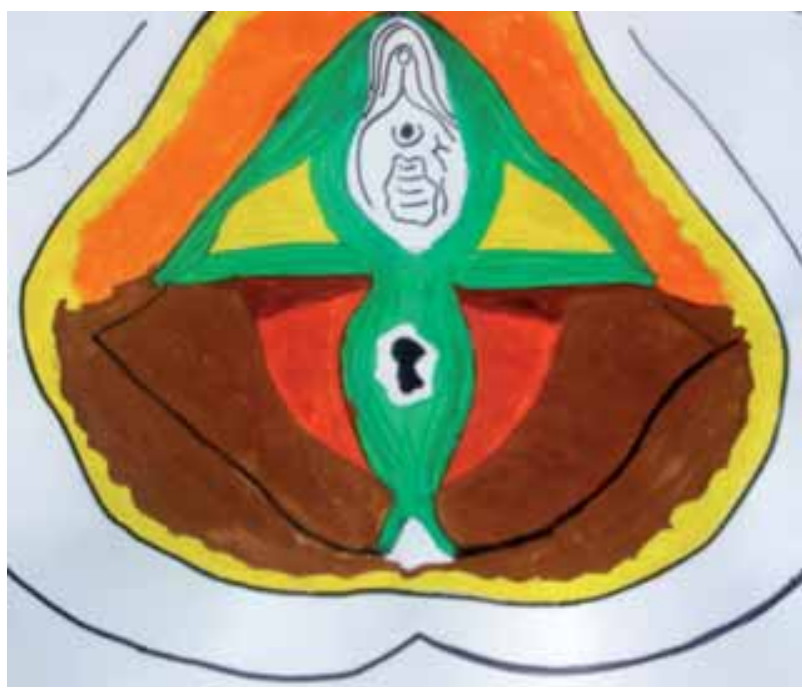

FIGURA 1. Músculos del suelo pélvico.

Fundamentados en la cinesiterapia o terapia del movimiento, se elaboran protocolos que consideran los tiempos de contracción y relajación apropiados para la trofia y fortalecimiento de los músculos y los ligamentos del suelo pélvico. Estos protocolos logran una reordenación fibrilar muscular, actúan sobre la plasticidad colágena (los músculos elevadores del ano son ricos en colágeno) y se consigue el restablecimiento de la funcionalidad del sistema de continencia.

\section{PACIENTES Y MÉTODOS}

\section{Pacientes}

El estudio se llevó a cabo en 85 mujeres de edad comprendida entre 42 y 74 años, diagnosticadas de IUE pura. El periodo de estudio y tratamiento fue desde Septiembre de 2002 hasta Junio de 2007. Las pacientes se dividieron en dos grupos: Grupo 1 $(n=50)$ : Pacientes con IUE tratadas mediante biofeedback (BFB) perineal con electrodos de superficie sin electroestimulación, dos sesiones semanales, de 30 minutos cada una, durante 10 semanas; y Grupo $2(\mathrm{n}=35)$ : Pacientes con IUE tratadas mediante reeducación de la musculatura de suelo pélvico (Kegel tipo 1) y electroestimulación vaginal, dos sesiones a la semana durante 10 semanas.

\section{Infraestructura}

Se utilizaron un equipo de Medicina y Mercado, modelo Solar Gold, para realizar el procedimiento de biofeebback con electrodos de superficie, y un equipo Enraf Nonus serie 9, modelo Myomed 932, para realizar la electroestimulación vaginal. 


\section{Procedimiento}

Las pacientes eran remitidas con un historial clínico donde se recogía la anamnesis, exploración física general, y estudios complementarios de rutina (bioquímica sanguínea, hemograma, sistemático de orina, urocultivo, y ecografía/citología de orina/cistoscopia en los casos de microhematuria). No se incluyeron en el estudio aquellas pacientes que presentaban trastornos psiquiátricos, de conducta, cognitivos o deformidades anatómicas congénitas, que pudieran afectar el resultado del tratamiento. Las pacientes fueron informadas de los beneficios esperados, riesgos y opciones de tratamiento, y firmaron un consentimiento informado para su participación en el estudio. Las pacientes completaron los cuestionarios incluidos en el estudio (IU-5, el ICIQ - SF y el cuestionario de salud King's), comprendiendo y dando el visto bueno a la agenda establecida, con el compromiso de acudir a consulta en las fechas señaladas.

El tratamiento se realizaba en posición de decúbito supino, con ligera flexión de cadera y protección de la lordosis lumbar para evitar sobrecarga. Desde esta posición podían establecer contacto visual con la pantalla del aparato para realizar el biofeedback o para recibir la electroestimulación. Previa explicación de la anatomía y fisiología del suelo pélvico se daba la orden a la paciente de realizar varias contracciones de la musculatura perineal de las cuales se hacía un registro en el que quedaba reflejada la potencia y tono muscular, así como los tiempos de contracción, según la electromiografía de superficie.

En el Grupo 1 cada paciente realizó un programa secuencial de tratamiento con biofeedback sobre la musculatura perineal, guiada por el terapeuta o monitor, desde zona perianal a zona periuretral, a partir de 3 segundos de contracción/5 segundos de relajación, durante 30 minutos, dos veces a la semana, con incremento hasta 5 segundos de contracción/10 segundos de relajación. El voltaje de la EMG fue aumentando desde $10 \mathrm{mV}$ hasta $25 \mathrm{mV}$ (Figs. 2, 3, 4 y 5).

En el Grupo 2 cada paciente realizó un programa secuencial de tratamiento con reeducación de músculos del suelo pélvico mediante ejercicios de Kegel tipo $1^{8-9}$ y electroestimulación vaginal (Fig.6). La electroestimulación vaginal fue llevada a cabo en dos sesiones a la semana durante 10 semanas. Los ejercicios de Kegel tipo 1 consisten en:

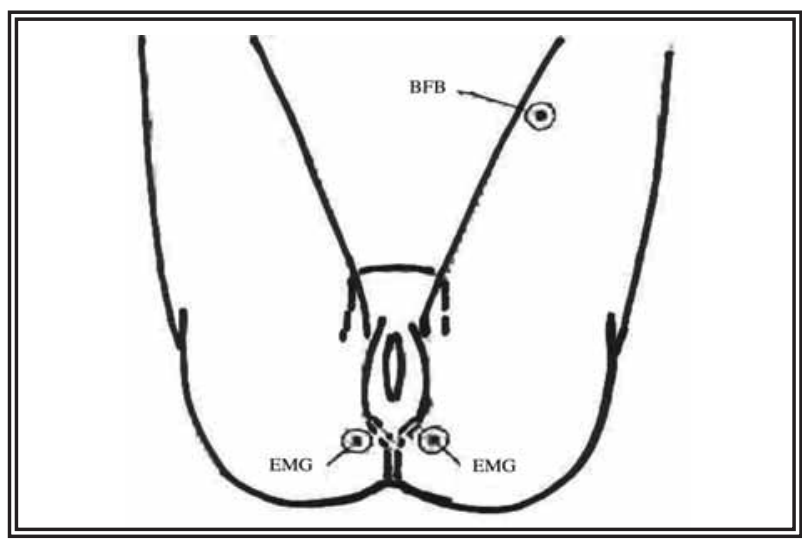

FIGURA 2. Colocación de electrodos.

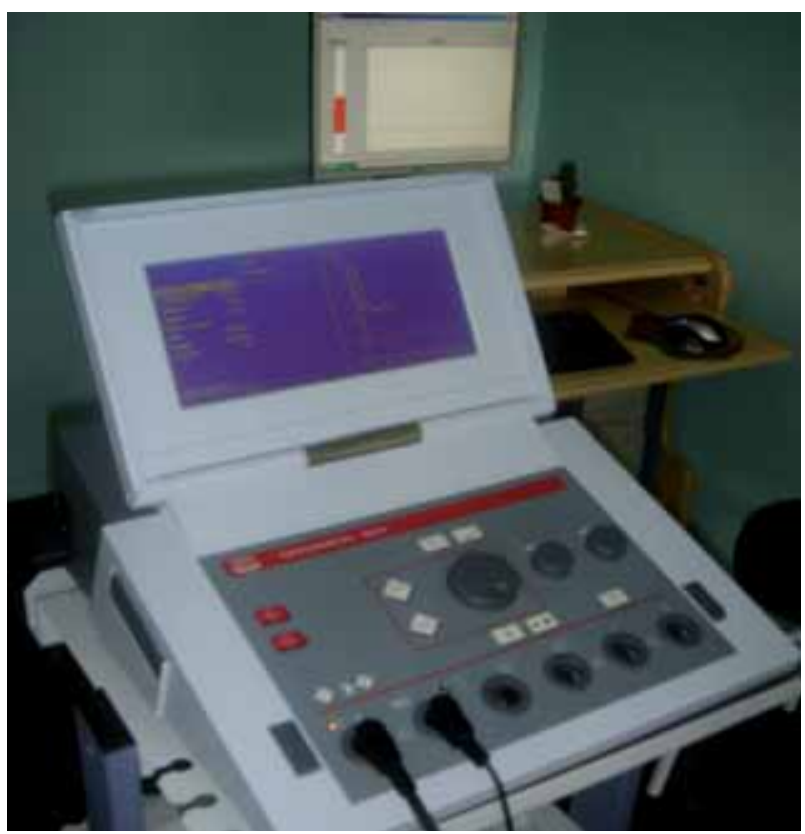

FIGURA 3. Pantalla de monitor para BFB sin escena (Enraf Nonus ${ }^{T M}$ ).

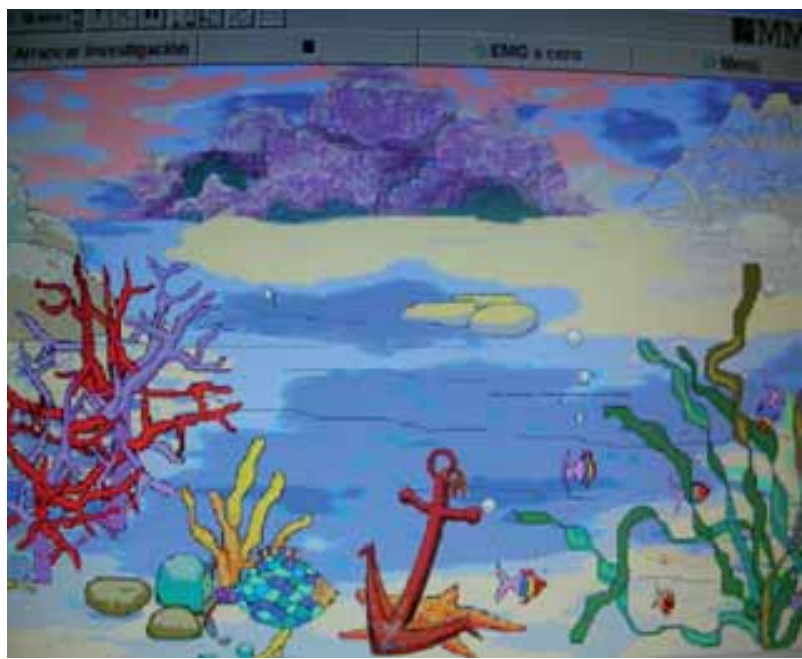

FIGURA 4. Pantalla de monitor para BFB con escena (Medicina y Mercado $^{T M}$ ). 


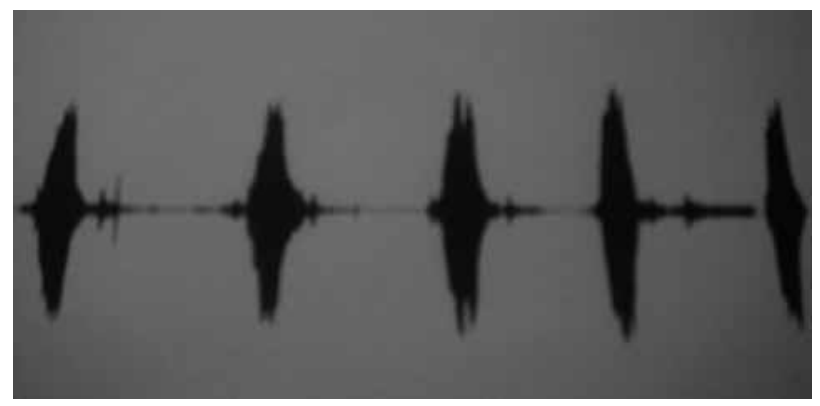

FIGURA 5. Registro EMG de BFB.

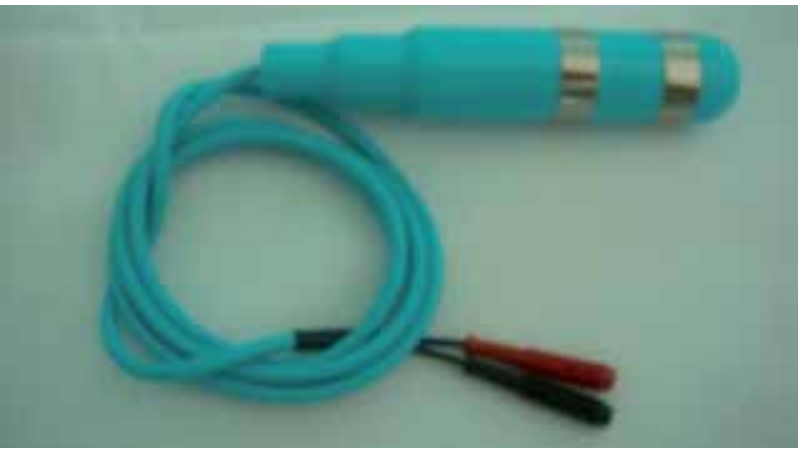

FIGURA 6. Electrodo para electroestimulación vaginal.

Variables controladas.

El número total de episodios o eventos, signos, tales como número de micciones, escapes de orina, uso de material protector (salvaslip, compresas, paños), cantidad de material protector usado y evaluación del uso del mismo (retirada húmedo, empapado, o ha resultado insuficiente causando traspaso a la ropa interior de la paciente), síntomas y alteración de la calidad de vida causada por los mismos, se han recogido en las encuestas estandarizadas que las pacientes respondieron. El análisis de la respuesta al tratamiento se realizó mediante estas encuestas, llevadas a cabo con una periodicidad semanal, contrastando los resultados con los hallazgos del registro EMG en el BFB. Las encuestas utilizadas son cuestionarios validados internacionalmente (IU-5) ${ }^{10-11}$ el ICIQ - SF ${ }^{12}$ y el cuestionario de salud King ${ }^{13}{ }^{13}$. Por lo tanto, se analizaron el número de episodios de IUE, el grado de necesidad de usar protección por IUE y la afectación de la calidad de vida por la IU.

Las edades de ambos grupos de estudio fueron homogéneas $(p=0,3203)$. La media de edad del Grupo 1 fue 49,67 años, SD 14,43. La media de edad del Grupo 2 fue 53,35 años, SD 15,30.

En las Tablas 1 y 2 se muestran los resultados obtenidos en el índice de incontinencia urinaria uti-
Tabla 1

\begin{tabular}{|c|c|c|c|}
\hline $\begin{array}{l}\text { Pacientes } \\
\text { del } \\
\text { Grupo } 2 \\
(\mathrm{~N}=35)\end{array}$ & $\begin{array}{l}\text { Valor basal en } \\
\text { cuestionarios } \\
\text { King's y en } \\
\text { ICIQ - SF } \\
\text { respectivamente }\end{array}$ & $\begin{array}{l}\text { Valor en } \\
\text { semana } 4 \text { en } \\
\text { cuestionarios } \\
\text { King's y en } \\
\text { ICIQ - SF } \\
\text { respectivamente }\end{array}$ & $\begin{array}{l}\text { Valor en } \\
\text { semana } 10 \text { en } \\
\text { cuestionarios } \\
\text { King's y en } \\
\text { ICIQ - SF } \\
\text { respectivamente }\end{array}$ \\
\hline 1 & $60-17$ & $22-2$ & $22-1$ \\
\hline 2 & $66-15$ & $38-6$ & $22-1$ \\
\hline 3 & 73-19 & $62-17$ & $61-16$ \\
\hline 4 & $58-15$ & $38-10$ & 23-2 \\
\hline 5 & 57-18 & $32-6$ & $19-1$ \\
\hline 6 & 64-17 & $38-9$ & $25-1$ \\
\hline 7 & 59-14 & $22-1$ & $22-1$ \\
\hline 8 & $70-17$ & $39-6$ & 25-1 \\
\hline 9 & $59-17$ & $36-5$ & $22-1$ \\
\hline 10 & $79-20$ & $69-17$ & 65-16 \\
\hline 11 & 64-17 & 54-14 & $25-2$ \\
\hline 12 & $56-17$ & $36-7$ & $22-2$ \\
\hline 13 & 58-15 & $31-5$ & $22-2$ \\
\hline 14 & $59-17$ & $37-6$ & $22-1$ \\
\hline 15 & $68-17$ & $43-7$ & $24-2$ \\
\hline 16 & 57-15 & $36-9$ & $22-2$ \\
\hline 17 & $57-15$ & $35-7$ & $22-2$ \\
\hline 18 & $61-17$ & $32-5$ & $22-1$ \\
\hline 19 & $60-15$ & $36-8$ & $22-1$ \\
\hline 20 & $65-15$ & $35-8$ & $22-1$ \\
\hline 21 & $78-20$ & $70-17$ & 69-17 \\
\hline 22 & $60-17$ & $37-7$ & $22-2$ \\
\hline 23 & $62-17$ & $60-16$ & $22-2$ \\
\hline 24 & $76-20$ & $70-17$ & 69-16 \\
\hline 25 & 64-17 & $36-6$ & $22-2$ \\
\hline 26 & $60-17$ & $30-8$ & $22-2$ \\
\hline 27 & $78-20$ & 70-19 & $69-17$ \\
\hline 28 & $86-19$ & $73-17$ & $67-17$ \\
\hline 29 & $62-17$ & $28-6$ & $22-1$ \\
\hline 30 & 64-15 & $35-5$ & $22-2$ \\
\hline 31 & $61-17$ & $27-8$ & $22-1$ \\
\hline 32 & $62-15$ & $34-8$ & 23-1 \\
\hline 33 & $61-17$ & $55-16$ & $23-2$ \\
\hline 34 & 75-18 & $67-17$ & 69-14 \\
\hline 35 & $63-17$ & $29-5$ & $22-1$ \\
\hline
\end{tabular}

lizando el cuestionario de salud King's y en el índice ICIQ - SF, expresados como valor basal al comienzo del estudio y los valores obtenidos en dichos cuestionarios en las semanas 4 y 10 durante el tratamiento, para los Grupos 1 y 2 respectivamente.

En el Grupo 1, el 50\% de las pacientes mejoraron en la semana cuatro y el $84 \%$ en la semana diez. En el Grupo 2, el 71,42\% de las pacientes mejoraron en la semana cuatro y el $80 \%$ en la semana diez. 
Tabla 2

\begin{tabular}{|c|c|c|c|}
\hline $\begin{array}{l}\text { Pacientes } \\
\text { del } \\
\text { Grupo } 1 \\
(\mathrm{~N}=50)\end{array}$ & $\begin{array}{l}\text { Valor basal en } \\
\text { cuestionarios } \\
\text { King's y en } \\
\text { ICIQ - SF } \\
\text { respectivamente }\end{array}$ & $\begin{array}{l}\text { Valor en } \\
\text { semana } 4 \text { en } \\
\text { cuestionarios } \\
\text { King's y en } \\
\text { ICIQ - SF } \\
\text { respectivamente }\end{array}$ & $\begin{array}{l}\text { Valor en } \\
\text { semana } 10 \text { en } \\
\text { cuestionarios } \\
\text { King's y en } \\
\text { ICIQ - SF } \\
\text { respectivamente }\end{array}$ \\
\hline 1 & $80-16$ & $40-5$ & $21-2$ \\
\hline 2 & $76-17$ & $77-17$ & $72-16$ \\
\hline 3 & $80-17$ & $31-6$ & $21-3$ \\
\hline 4 & $45-12$ & $21-2$ & $21-2$ \\
\hline 5 & 71-16 & $36-8$ & $24-2$ \\
\hline 6 & $90-15$ & $90-13$ & $31-1$ \\
\hline 7 & $49-17$ & $49-12$ & $21-2$ \\
\hline 8 & $70-11$ & $24-2$ & $24-1$ \\
\hline 9 & 70-19 & $49-5$ & $24-2$ \\
\hline 10 & $56-12$ & $24-2$ & $24-1$ \\
\hline 11 & $56-14$ & $24-5$ & $21-2$ \\
\hline 12 & $77-15$ & $49-6$ & 24-1 \\
\hline 13 & $91-21$ & $79-17$ & $79-17$ \\
\hline 14 & $50-14$ & $25-2$ & $24-2$ \\
\hline 15 & 71-15 & $25-2$ & 21-1 \\
\hline 16 & 71-16 & 71-16 & $22-2$ \\
\hline 17 & $62-14$ & $62-14$ & $22-2$ \\
\hline 18 & 79-14 & 79-14 & $22-2$ \\
\hline 19 & $46-11$ & $46-11$ & $21-1$ \\
\hline 20 & 73-14 & $21-2$ & 21-1 \\
\hline 21 & $86-16$ & $22-3$ & 24-1 \\
\hline 22 & $62-15$ & $62-14$ & $62-14$ \\
\hline 23 & $68-16$ & $68-14$ & 24-1 \\
\hline 24 & $72-15$ & $72-15$ & $22-2$ \\
\hline 25 & $78-14$ & $22-2$ & $22-1$ \\
\hline 26 & $86-16$ & $39-6$ & $24-2$ \\
\hline 27 & $61-15$ & $41-3$ & $22-1$ \\
\hline 28 & $48-14$ & $30-3$ & $22-1$ \\
\hline 29 & $87-17$ & $71-15$ & 71-14 \\
\hline 30 & $66-15$ & $32-2$ & $22-1$ \\
\hline 31 & $78-18$ & $70-14$ & 69-13 \\
\hline 32 & $56-13$ & $37-6$ & 23-1 \\
\hline 33 & $53-16$ & $32-5$ & 25-1 \\
\hline 34 & $58-15$ & $41-5$ & $22-1$ \\
\hline 35 & 69-17 & $38-6$ & 23-1 \\
\hline 36 & $76-21$ & 68-17 & $30-4$ \\
\hline 37 & $60-14$ & $30-6$ & $22-1$ \\
\hline 38 & $48-13$ & $22-2$ & $22-1$ \\
\hline 39 & $70-14$ & $22-1$ & $22-1$ \\
\hline 40 & $82-20$ & $69-17$ & 69-17 \\
\hline 41 & $62-17$ & $61-14$ & $22-1$ \\
\hline 42 & 69-17 & 69-16 & $22-2$ \\
\hline 43 & $48-14$ & $48-13$ & $22-1$ \\
\hline 44 & $67-14$ & $67-14$ & $22-2$ \\
\hline 45 & $86-17$ & $72-17$ & $72-16$ \\
\hline 46 & $72-16$ & $72-17$ & $72-17$ \\
\hline 47 & $66-17$ & $66-17$ & $24-2$ \\
\hline 48 & $60-14$ & $60-14$ & $22-1$ \\
\hline 49 & $78-17$ & $73-16$ & $22-2$ \\
\hline 50 & $64-13$ & $64-13$ & $22-1$ \\
\hline
\end{tabular}

En el Grupo 2, en 5 de las 28 pacientes que sí mejoraron y en 3 de las 7 que continuaron incontinentes, aparecieron efectos secundarios locales (irritación vaginal, dispareunia), que en ningún caso ocasionó abandono del tratamiento aunque sí tratamiento sintomático. Los resultados en el cuestionario de calidad de vida debida a síntomas urinarios se correlacionaron de forma directa positiva con la mejoría debida al tratamiento en todas las pacientes.

No hubo diferencia estadísticamente significativa entre ambos grupos de tratamiento en cuanto a conseguir la curación de la incontinencia $(\mathrm{p}=0,1265)$ ni en la en la mejoría de la calidad de vida debida a sintomas urinarios $(\mathrm{p}=0,3217)$.

\section{DISCUSIÓN}

El tratamiento de la incontinencia urinaria continúa siendo un reto en nuestros días. Es un problema muy frecuente $y$, aunque manejamos cifras imprecisas, en nuestro medio hasta el 37\% de mujeres de entre 35 y 54 años y más del 40\% de las mayores de 55 años, precisan tratamiento.

Entre las alternativas terapéuticas para la IU está el tratamiento conservador, mediante fármacos, o mediante el tratamiento del comportamiento, el tratamiento quirúrgico y, a caballo entre ambas, un procedimiento mínimamente invasivo, que es la neuroestimulación, útil en casos de incontinencia en la disfunción miccional crónica en vejigas neurógenas, entre otros.

Respecto al tratamiento quirúrgico, se ha de considerar que las alteraciones anatómicas y disfunciones del suelo pélvico femenino implican simultáneamente a más de un órgano o aparato hasta en un 61 por ciento de los $\operatorname{casos}^{14}$. Por lo tanto, un abordaje multidisciplinar coordinado en este tipo de patología, tanto en el aspecto diagnóstico como terapéutico, parece el más apropiado, aunando el conocimiento más exhaustivo y especializado de la Urología, la Ginecología y la Cirugía ${ }^{14}$.

El análisis de los resultados de la cirugía en el control al sexto mes tras la intervención, arroja cifras de mejoría del 95\% en la valoración subjetiva por parte de las pacientes. Sin embargo, de manera objetiva, se consideraron totalmente asintomáticas sólo un 57\%. Se reintervienen hasta el 8,20\% de pacientes y la morbilidad sistémica y/o mortalidad comunicadas son del $0 \%{ }^{14}$. 
Hasta ahora, la cirugía se basaba en la reconstrucción de la estructura muscular con los propios tejidos dañados. Los nuevos "slings" o cabestrillos y mallas artificiales de polipropileno son tolerados por el organismo y ofrecen buenos resultados en casos seleccionados. El tratamiento con banda libre de tensión se considera actualmente el de primera elección en la incontinencia de esfuerzo de moderada a severa ${ }^{15}$.

La incrustación de la malla de polipropileno intravesical con infección del tracto urinario crónica, la erosión de la malla en la mucosa vaginal a través de la incisión vaginal, la erosión uretral y la retención urinaria permanente por obstrucción del tracto de salida son, entre otras, algunas de las complicaciones comunicadas. En estos casos, los síntomas pueden ser urgencia, incontinencia de urgencia y hematuria macroscópica. Algunas pacientes con erosión uretral, erosión vaginal y/o de la vejiga, y las que presentan obstrucción del tracto de salida, precisan retirar el cabestrillo y cirugía posterior para reestablecer la continencia ${ }^{16}$.

En los casos de recurrencia de la IU, puede repetirse el procedimiento quirúrgico y se puede colocar un nuevo cabestrillo. Las complicaciones con procedimientos con TVT (Tension Vaginal Tape) que requieren una intervención quirúrgica adicional, aumentan la morbilidad significativamente ${ }^{17}$.

Dentro de los "foros quirúrgicos", se reconoce que la cirugía no es la única solución a la incontinencia urinaria, y que, por ejemplo tras los partos, es fundamental la prevención de la IU mediante ejercicios de rehabilitación y fortalecimiento ${ }^{18}$.

\section{Tratamiento del comportamiento o "tratamiento}

funcional de la IU"

Durante muchos años el tratamiento de la IU se ha contemplado bajo dos únicos puntos de vista: el farmacológico y el quirúrgico ${ }^{19}$. Posiblemente esta limitación ha estado condicionada por la no disponibilidad de instalaciones, aparataje o tiempo para llevar a cabo un tratamiento funcional.

Actualmente, existe consenso acerca de que el tratamiento inicial de la IUE femenina de grado leve y moderado debe ser siempre conservador (fármacos o tratamiento funcional del suelo pélvico) y sólo si no hay respuesta se plantea la cirugía. El tratamiento funcional del suelo pélvico en la IU incluye la reeducación de hábitos miccionales; el entrenamiento vesical; y los ejercicios de suelo pélvico, mediante biofeedback u otra técnica ${ }^{15}$.
La continencia urinaria en la mujer depende fundamentalmente de la posición y movilidad de la uretra y de la unión uretrovesical. Los músculos del suelo pelviano (MSP) y la fascia endopélvica son los elementos fundamentales para mantener la posición y movilidad correctas de la uretra. Durante los esfuerzos los MSP incrementan la resistencia. Una contracción eficaz de los MSP puede comprimir la uretra contra la sínfisis del pubis, aumentando la presión intrauretral $\mathrm{y}$, por consiguiente, su resistencia a la salida involuntaria de orina. El efecto en la presión uretral de la contracción voluntaria de los MSP se ha demostrado mediante estudios urodinámicos ${ }^{20}$.

La reeducación de los músculos del suelo pelviano (RMSP) es el tratamiento conservador más utilizado para las mujeres con síntomas de IUE. Consiste en realizar contracciones voluntarias repetidas de estos músculos. Puede ayudarse con técnicas de biofeedback y/o electroestimulación (EE). Se han publicado una gran variedad de programas de entrenamiento muscular. Existe evidencia científica suficiente como para recomendar el tratamiento con RMSP en mujeres no sólo con IUE sino también IU mixta ${ }^{20}$.

Se ha demostrado que los programas de RMSP mejoran el tono muscular en reposo y la coordinación en la contracción muscular para estabilizar la uretra y mantener la continencia durante los esfuerzos ${ }^{20}$. Lo principal para realizar el tratamiento con RMSP es que la mujer tome conciencia de la contracción y aprenda a realizarla de manera correcta ${ }^{21}$.

Se ha estudiado el conocimiento que las mujeres tienen de los MSP y la capacidad de contraerlos después de una explicación teórica sobre cómo hacerlo ${ }^{22}$. Monitorizando el resultado con tacto vaginal y perineometría, se ha observado que después de la instrucción verbal sólo el $68 \%$ de las mujeres son capaces de realizar una contracción correcta. Otros estudios comunican que sólo el $51 \%$ de las mujeres son capaces de contraer los músculos del suelo pelviano después de una instrucción verbal por un profesional ${ }^{23}$. Así pues, antes de iniciar un programa de RMSP es imprescindible evaluar la capacidad que tiene la mujer de realizar una contracción voluntaria de estos músculos de manera eficaz. La capacidad contráctil de los MSP se puede evaluar mediante palpación vaginal (testing muscular), perineometría y electromiografía ${ }^{20}$.

Las técnicas de biofeedback consisten en recoger los signos biológicos del organismo y traducirlos en una señal sensitiva perceptible que cambia de intensidad según las propias variaciones del sistema biológico analizado ${ }^{19}$. 
El equilibrio entre las fases de llenado y vaciado del ciclo miccional es modulado por los circuitos de integración cortical, los arcos reflejos sacros y la musculatura del suelo pélvico [24]. Cualquier deficiencia o alteración en algún elemento de la cadena puede dar lugar a una disfunción miccional y alteración del mecanismo de continencia ${ }^{19}$.

El papel de la musculatura del suelo pélvico en este equilibrio es fundamental. El suelo pélvico participa en el ciclo miccional iniciando o interrumpiendo éste, bajo control cortical. La relajación voluntaria mediada corticalmente y vehiculizada por medio del nervio pudendo es fundamental para iniciar la fase de vaciado, con la consiguiente activación refleja del sistema parasimpático (nervio pélvico) que conlleva la contracción del detrusor, y la inhibición simpática (nervio hipogástrico) que relaja el cuello vesical, consiguiendo de esta forma una micción normalmente coordinada. Por otra parte, en la fase de llenado, la contracción mantenida del suelo pélvico inhibe de forma refleja la contracción del detrusor, perpetuándose el tono simpático que mantiene el cuello vesical cerrado, a la vez que aumenta el tono de la musculatura periuretral, requisitos necesarios para la continencia en esta fase, donde se precisa la estabilidad del detrusor y altas resistencias uretrales ${ }^{19}$.

El componente consciente del ciclo miccional, la musculatura periuretral, puede aumentar su contracción o aprender a relajarse y de esta forma influir en dicho balance, restableciendo el ciclo miccional adecuado. Este es el principio de las técnicas conservadoras de reeducación miccional. El restablecer el balance alterado, normalizará el ciclo miccional en patologias tan opuestas como la incontinencia por urgencia o el retencionista crónico ${ }^{25}$.

Los equipos más completos de $\mathrm{BFB}$, como los utilizados en el presente estudio, disponen de dos canales: un canal registra la actividad del suelo pélvico (electrodos perineales o vaginales), y el otro registra la actividad de grupos musculares antagonistas o "control" (electrodos de superficie abdominales o en el muslo). La información que dispone el paciente es mucho más completa, de forma que puede contraer o relajar específicamente un grupo muscular concreto, observando si realiza incorrectamente el ejercicio (utilizando los músculos abdominales o de la extremidad inferior en lugar de los perineales). La realización inadecuada de los ejercicios no sólo no conseguirá mejoría clínica, sino que la empeorará con el fortalecimiento de músculos antagonistas ${ }^{26}$. El biofeedback, visual o de otro tipo (auditivo, flujometría) ayuda a la realización correcta de los ejercicios y es especialmente útil en aquellos pacientes que tienen dificultades en la localización de la musculatura perineal. Desde hace más de veinticinco años se han comunicado resultados de curación o mejoría mediante biofeedback vesico-esfinteriano ${ }^{27-28 .}$ A pesar de ello, no son "obligatoriamente necesarias" las unidades de tratamiento de suelo pelviano en todos los hospitales generales de nuestro país. Puede deberse al tiempo que se precisa para desarrollar una unidad eficaz que lleve a cabo tratamientos de relativamente larga duración (entre 2 y 4 meses en cada paciente) frente a la presión asistencial. Aunque hay diversos protocolos publicados (20minutos 3 veces a la semana durante 7 semanas $^{29,30}, 30$ minutos dos veces a la semana durante 6 semanas $^{29}$; 12 sesiones semanales ${ }^{31}$; 2 sesiones a la semana durante 6 semanas $^{32}$ ) los sistemas sanitarios más avanzados de diversos países europeos, admiten como primera elección para el tratamiento conservador de la IUE el tratamiento funcional de los músculos del suelo pélvico, cuyas opciones preponderantes son el biofeedback, la estimulación eléctrica y los conos vaginales entre otros ${ }^{33}$.

Según la experiencia aportada por unidades funcionales de suelo pelviano en Estados Unidos, y apoyados en la evidencia científica aportada por expertos en este tratamiento ${ }^{29}$, creemos que lo importante en el tratamiento funcional del suelo pelviano son los protocolos rigurosos, terapeuta guiados, focalizando la actividad en la musculatura perineal superficial. El terapeuta o monitor puede ser personal sanitario de diversa categoría pero con entrenamiento específico en estos protocolos, donde la paciencia y el manejo psicosomático del paciente son cruciales. Hemos encontrado, al igual que otros autores ${ }^{29}$, que los resultados utilizando electrodos sin electroestimulación son mejores que los obtenidos con electroestimulación. Esto se ha demostrado tanto con electroestimulación superficial como con electroestimulación intravaginal ${ }^{34}$, de forma que tanto el número de micciones en 24 horas como la satisfacción obtenida con el tratamiento fue significativamente superior en las mujeres tratadas sin electroestimulación. La IU de urgencia se reduce incluso más en las pacientes sometidas a un protocolo riguroso sin electroestimulación (31\% de reducción de episodios de IU de urgencia frente a $15 \%$ de reducción de episodios en mujeres con electroestimulación. Aunque en la evaluación subjetiva parece que hay diferencias en la mejoría en la IU de urgencia a favor de pacientes tratadas con electroestimulación, la evaluación objetiva utilizando 
perineómetro no ha arrojado diferencias estadísticamente significativas entre grupos tratados con y sin electroestimulación, encontrando en todas las pacientes una mejoría importante con el tratamiento. Igualmente ocurre con el test de peso vaginal: no hay diferencias en la mejoría encontrada en las pacientes tratadas con y sin electroestimulación ${ }^{34}$.

Otro aspecto importante es la evidencia de la superioridad de un programa protocolizado terapeuta/ monitor guiado frente a aparatos de uso domiciliario controlados por el propio paciente. Se evaluó la eficacia de la electroestimulación en mujeres con IUE, IU de urgencia y cistopatía intersticial utilizando un electroestimulador portátil. El ajuste de la electroestimulación se hizo en relación a la sensación del paciente. El uso de aparatos ambulatorios, incluso con mecanismos finos y avanzados, como los ideados para la electroestimulación de los músculos perineales a demanda, en caso de la IUE cuando aumenta la presión abdominal, o para la IU de urgencia, o incluso para patología como la cistopatía intersticial, no ha demostrado ventajas sobre los protocolos terapeuta/monitor guiados en centros especializados y muy experimentados en estos tratamientos ${ }^{35}$.

\section{CONCLUSIONES}

Los resultados exitosos del tratamiento con un programa de biofeedback perineal con electrodos de superficie o de reeducación de los músculos del suelo pélvico más electroestimulación vaginal, en la incontinencia urinaria de esfuerzo leve y moderada, son similares o superiores a los publicados en tratamientos más invasivos.

Ambos protocolos son inocuos, aunque ocasionalmente la electroestimulación vaginal puede ocasionar molestias locales.

\section{REFERENCIAS}

1. Abrahams PJ, Blaivas S. Stanton, The standarization of terminology of lower urinary tract function. Scan J Urol Nephrol. 1988;114-5.

2. Blaivas JG, Olsson CA. Stress incontinence: classification and surgical approach. J Urol. 1988;139(4):727-731.

3. Artibani W. Un tercio de las mujeres padece incontinencia urinaria de esfuerzo. Diario Médico, 2003.

4. Castro D. The pelvic floor functional anatomy. in The pelvic floor functional anatomy. Pelvic Floor Reconstruction. 2002. Murcia. España: European School of Urology.

5. Netter, F., Sistema reproductor., in Colección Ciba de ilustraciones médicas. 1979, Salvat: Mexico.

6. Netter, F., Atlas de Anatomía Humana. 1999: Novartis-Masson.

7. Petros PE, Ulmsten UI. An integral theory and its method for the diagnosis and management of female urinary incontinence. Scan J Urol Nephrol, 1993;153:1-93.

8. Kegel A., Progressive resistance exercise in the functional restoration of the perineal muscles. An J Obst Gyn. 1948;56: 238-248.

9. Kegel AH. Physiologic therapy for urinary stress incontinence. J Am Med Assoc. 1951;146(10):915-917.

10. McFall S, Yerkes AM, Bernard M, LeRud T. Evaluation and treatment of urinary incontinence. Arch Fam Med. 1997:6(2):114-119.
11. Viktrup, L., K. Summers, and S. Dennett, Clinical Urology Guidelines for the Initial Assessment and Treatment of Women with Urinary Incontinence: A Review. European Urology Supplements, 2005;4(1):38-45.

12. Espuña Pons M, Rebollo Alvarez P, Puig Clota M. Validación de la versión española del ICIQ-SF. Un cuestionario para evaluar la incontinencia urinaria. Med Clin. 2004; 122(8):288-292

13. López-Carrión, M., ¿Podemos mejorar el manejo de la incontinencia urinaria? Pautas de actuación., in Enfermedades nefro-urológicas, R. Medynet, Editor. 2005, Medynet: Madrid.

14. Pacanowski, T., La cirugía en una sesión puede ser óptima en el suelo pélvico., in Sección Cirugía General, D. Médico.com, Editor. 2002, Recoletos.es: Barcelona. p. http://buscador.recoletos.es/advice/pruebas/documento.jsp.

15. Serrano, R., El $90 \%$ de las IU de esfuerzo se evitan con medidas preventivas., in Urologia, D. Médico.com, Editor. 2003, Recoletos.es: Madrid. p. http://www.diariomedico.com /edicion/noticia/0,2458,404697,00.html.

16. Sweat SD, Itano NB, Clemens JQ, Bushman W, Gruenenfelder J, McGuire EJ, et al., Polypropylene Mesh Tape for Stress Urinary Incontinence: Complications of Urethral Erosion and Outlet Obstruction. J Urol. 2002;168(1):144-146.

17. Volkmer, B., et al., Surgical intervention for complications of tension-free vaginal tape procedure. J Urol, 2003. 169(2): p. 570-4.

18. Serrano, C., Las mallas artificiales de polipropileno reparan fallos de suelo pélvico., in Especialidades, Diariomédico.com, Editor. 2003, Recoletos.es: Madrid. http://www.diariomedico.com.

19. Arlandis-Guzmán, S., E. Martínez-Agulló, and J. Jiménez-Cruz, Alternativas terapéuticas para la disfunción miccional crónica., in Neuromodulación: Una nueva alternativa terapéutica para los tratornos del tracto urinario inferior, A.E.d. Urologia, Editor. 2002, ENE Ediciones: Madrid. p. Cap 5.

20. Espuña-Pons, M., Criterios para la indicación de tratamiento conservador de la incontinencia urinaria de esfuerzo y tipos de tratamiento., in La Opinión de los expertos, E. Glosa, Editor. 2002, Edit Glosa: Barcelona. p. 12-14.

21. Bø K, Talseth T. Change in urethral pressure during voluntary pelvic floor muscle contraction and vaginal electrical stimulation. Int Urogynecol $\mathrm{J}$ Pelvic Floor Dysfunct. 1997;8(1):3-6.

22. Bo K, et al. Knowledge about and ability to correct pelvic floor muscle exercise in women with urinary stress incontinence. Neurol Urodyn, 1988. 7: p. 261-2.

23. Bump RC, Hurt WG, Fantl JA, Wyman JF. Assessment of Kegel pelvic muscle exercise performance after brief verbal instruction. Am J Obstet Gynecol. 1991;165(2):322328

24. Martínez-Agulló, E., J. Gallego, and J. Ruiz, Neurofisiología de la micción., in Incontinencia Urinaria. Conceptos actuales, E. Martínez-Agulló, Editor. 1990, Indas: Madrid. p. 53-70.

25. González-Chamorro, f., E. Lledó, and C. Llorente, La Neuromodulación como tratamiento de la disfunción miccional. Revisiones en Urología, 2001. II(1): p. 26

26. Llorca-Miravet, A., Tratamiento funcional en la incontinencia urinaria., in Incontinencia Urinaria: Conceptos Actuales, E. Martínez-Agulló, Editor. 1990, Graficuatre: Valencia. p. 629-649.

27. Sugar EC, Firlit CF. Urodynamic biofeedback: a new therapeutic approach for childhood incontinence/infection (vesical voluntary sphincter dyssynergia). J Urol. 1982;128(6): 1253-1258.

28. Rapariz M. Reeducación esfinteriana en el tratamiento de la Micción no Coordinada, in Departamento de Urologia. 1994, Universidad Complutense de Madrid: Madrid.

29. Amaro JL, Gameiro MO, Kawano PR, Padovani CR. Intravaginal electrical stimulation: a randomized, double-blind study on the treatment of mixed urinary incontinence. Acta Obstet Gynecol Scand. 2006;85(5)619-622.

30. Lee IS, Choi ES. Pelvic floor muscle exercise by biofeedback and electrical stimulation to reinforce the pelvic floor muscle after normal delivery. Taehan Kanho Hakhoe Chi. 2006;36(8):1374-1380.

31. Rett MT, Simoes JA, Herrmann V, Pinto CL, Marques AA, Morais SS. Management of stress urinary incontinence with surface electromyography-assisted biofeedback in women of reproductive age. Phys Ther. 2007;87(2):136-142.

32. Seo JT, Yoon H, Kim YH. A randomized prospective study comparing new vaginal cone and FES-Biofeedback. Yonsei Med J. 2004;45(5):879-884.

33. Jundt K, Friese K. Female urinary incontinence. MMW Fortschr Med. 2005;147(23) :26-29.

34. Amaro JL, Gameiro MO, Padovani CR. Effect of intravaginal electrical stimulation on pelvic floor muscle strength. Int Urogynecol J Pelvic Floor Dysfunct. 2005;16(5):355358

35. Nissenkorn I, Shalev M, Radziszewski P, Dobronski P, Borkowski A, De Jong PR. Patient-adjusted intermittent electrostimulation for treating stress and urge urinary incontinence. BJU Int. 2004;94(1):105-109.

Correspondencia autor: Dra. M.F. Lorenzo Gómez

Servicio y Cátedra de Urología.

Hospital Universitario de Salamanca. Universidad de Salamanca.

Po San Vicente, 58-152 - 37007 Salamanca. Tel.: 923291100

E-mail autor: mflorenzogo@yahoo.es

Información artículo: Original - Urología femenina

Trabajo recibido: septiembre 2007

Trabajo aceptado: noviembre 2007 